\title{
Sinopsis del género Opuntia Mill. (Opuntioideae, Cactaceae) en Honduras
}

\author{
Synopsis of the genus Opuntia Mill. (Opuntioideae, Cactaceae) \\ in Honduras
}

Olga P. Pineda ${ }^{1, \star}$ y Olvin W. Oyuela ${ }^{1}$

Recibido: 04 mayo 2020 | Aceptado: 29 julio 2020 | Publicado en línea: 01 agosto 2020

Citación: Pineda, OP; Oyuela, OW. 2020. Sinopsis del género Opuntia Mill. (Opuntioideae, Cactaceae) en Honduras. Revista Forestal del Perú 35(1): 54-72. DOI: http://dx.doi.org/10.21704/ $\underline{\text { rfp.v35il.1475 }}$

\begin{abstract}
Resumen
El género Opuntia, incluido en la sub familia Opuntioideae (Cactaceae), es uno de los géneros más complejos y diversos de la familia, y está caracterizado por sus tallos articulados en cladodios y presencia de gloquidios. Hasta la fecha, Honduras no cuenta con mucha información disponible respecto a la flora que posee, es por ello que, esta investigación realizó la actualización taxonómica del género Opuntia para el país. Para tal fin, se realizó una revisión de los ejemplares de Opuntia depositados en los herbarios Paul C. Standley (EAP) y Cyril Hardy Nelson Sutherland (TEFH) para obtener información sobre las especies de este género (sitios de recolecta, hábitat, fenología y nombres comunes). Este estudio reconoce ocho especies de Opuntia nativas para Honduras (O. deamii, O. decumbens, O. dejecta, O. guatemalensis, O. hondurensis, O. lutea, O. pubescens y O. velizii). El reconocer estas especies se debe en parte, por la conjunción de Nopalea en Opuntia y, por otro lado, por el registro de especímenes de herbario y de documentación precisa. Además, no se reconoce a O. tomentosa $(=$ O. tomentella) por la falta de ejemplares de herbario y documentación. Estas ocho especies están sujetas a CITES dentro del apéndice II y en la UICN solo tres especies se encuentran en la categoría LC (preocupación menor) y dos especies en la categoría DD (datos insuficientes).
\end{abstract}

Palabras clave: Neotrópico, Mesoamérica, taxonomía, cactos, nopales

\footnotetext{
${ }^{1}$ Herbario Cyril Hardy Nelson Sutherland (TEFH), Escuela de Biología, UNAH, Boulevar Suyapa, Tegucigalpa, Honduras.

* Autor de Correspondencia: olga.pineda@unah.edu.hn
} 


\begin{abstract}
The genus Opuntia, included in the sub family Opuntioideae (Cactaceae), is one of the most complex and diverse genera of the family, and it is isolated by its articulated stems in cladodes and the presence of glochids. To date, Honduras does not have much information available regarding the flora it possesses, which is why this research carried out the taxonomic update of the genus Opuntia for the country. To this end, a review of the examples of Opuntia deposited in the herbaria Paul C. Standley (EAP) and Cyril Hardy Nelson Sutherland (TEFH) was carried out to obtain information on the species of this genus (collection sites, habitat, phenology and common names). This study recognizes eight species of Opuntia native to Honduras (O. deamii, O. decumbens, O. dejacta, $O$. guatemalensis, $O$. hondurensis, O. lutea, O. pubescens and $O$. velizii). The recognition of these species is due, in part, by the conjunction of Nopalea in Opuntia and, on the other hand, by the registration of herbarium specimens and accurate documentation. Furthermore, O. tomentosa $(=O$. tomente$\mathrm{lla}$ ) is not recognized due to the lack of herbarium examples and documentation. These eight species are subject to CITES under Appendix II and at IUCN there are three species in the LC category (least concern) and two species in the DD category (insufficient data).
\end{abstract}

Key words: Neotropic, Mesoamerica, taxonomy, cactus, nopales

\section{Introducción}

La familia Cactaceae es un grupo de plantas eudicotiledóneas, perteneciente al orden Caryophyllales (APG IV 2016), es nativa del continente americano (Arias 2009; Bravo y Arias 2011). Se distribuye desde Canadá hasta la Patagonia en Argentina, el Caribe e Islas Galápagos (Ostolaza 2011; Pauca-Tanco y Quipuscoa-Silvestre 2017; Köhler et al. 2018), se encuentran en bosques secos hasta bosques húmedos, desde el nivel del mar hasta por encima de los 4000 m.s.n.m (Véliz 2008). Se especula que presenta cerca de 2360 especies incluyendo subespecies, distribuidas en 177 géneros (Lodé 2015; Bueno y Plumed 2017).

Los cactus son plantas que se caracterizan por poseer estructuras morfológicas propias, por ejemplo, las areolas, de las cuales se pueden desarrollar tallos, flores, espinas y/o gloquidios, y presentan un meristemo apical organizado en cuatro zonas distintivas, adicionalmente, los cactus tienen un ovario embebido en el receptáculo, conocido como pericarpelo (Fuentes-Perez et al. 2009; Arias 2009). Sus tallos son mayormente suculentos, verdes, ramificados, con formas globosas, cilíndricas y aplanadas, esta última forma conocida como cladodios. Las hojas por lo general están modificadas en espinas, con algunas excepciones (Bravo y Arias 2011). Las flores son hermafroditas, rara vez unisexuales, y mayormente solitarias, pero pueden estar agrupadas en inflorescencias como en el caso de Pereskia Mill. (Ostolaza 2011; Arias et al. 2012). Presentan frutos en bayas, con numerosas semillas con o sin arilo. Su hábito de crecimiento puede ser arbóreo, arbustivo, trepador o epífito (Arias y Véliz 2006; Vázquez et al. 2012).

Anteriormente, la familia Cactaceae estaba dividida en tres subfamilias: Pereskioideae, Opuntioideae y Cereoideae, esta división estuvo basada en características morfológicas (Mercado 2014). Sin embargo, las clasificaciones filogenéticas recientes dividen a Cactaceae en cuatro subfamilias: Pereskioideae, Opuntioideae, Maihuenioideae y Cactoideae (Vázquez et al. 2012, Arias et al. 2012). No obstante, algunos autores reconocen además a Leuenbergioideae como una quinta subfamilia (Stevens 2001 en adelante; Bueno y Plumed 2017; De Almeida et al. 2018). Respecto a la subfamilia Opuntioideae, es la segunda más extensa de la familia después de Cactioideae, e incluye cinco tribus Austrocylindropuntieae, Pterocacteae, Tephrocacteae, Cylindropuntieae y Opuntieae, siendo las dos últimas conocidas como nopales o tunas (Griffith y 
Sinopsis del género Opuntia Mill. (Opuntioideae,

Porter 2009; Mercado 2014). Dentro de la tribu Opuntieae se encuentra incluido el género Brasiliopuntia (K. Schum.) A. Berger, Consolea Lem., Miqueliopuntia Frič ex F. Ritter, Tacinga Britton \& Rose, Tunilla D.R. Hunt \& Iliff y Opuntia Mill., este último genero constituye uno de los grupos más grandes, diversos y complejos de esta familia (Espinosa y Vargas 2002; Fuentes-Pérez et al. 2009, Las Peñas et al. 2017; Köhler et al. 2018).

El género Opuntia se identifica por mostrar tallos fotosintéticos articulados en cladodios mayormente aplanados, cilíndricos o globosos, areolas con espinas, algunas espinas alargadas y finas llamadas cerdas; otras espinas defensivas conocidas como gloquidios o "ahuates", propias de la subfamilia Opuntioideae, estos son muy delgados, pequeños, rígidos, y muy numerosos (Bravo 1978). Las flores son hermafroditas, solitarias, terminales o subterminales, diurnas, deciduas, con gloquidios en sus areolas, a veces con cerdas o espinas; tubo receptácular corto; perianto en forma de roseta y tubular, rojos, anaranjados, rosados, amarillos o verdosos; múltiples estambres, ovario ínfero con varios óvulos; frutos en baya, rojos al madurar, ocasionalmente con gloquidios y con semillas ariladas (Bravo y Arias 2011; Köhler et al. 2018).

Muchas de las especies son variables por efecto de la dispersión natural, factores ambientales, la hibridación y el cultivo. Algunos autores creen que este género puede incluir 160 especies, a nivel del continente americano (Espinosa y Vargas 2002). Aunque, según Griffith y Porter (2009) hay 190 especies aceptadas y 36 en estudio, y asimismo Köhler et al. (2018) mencionan que existen alrededor de 150 especies de este género.

Honduras no cuenta con mucha información disponible respecto a la flora que posee, en relación a cactáceas, se estima alrededor de 40 especies entre nativas e introducidas (Shulze 2004). House y Midence (2007) reportan 45 especies de cactáceas nativas, mientras que Nelson (2008) reporta alrededor de 68 incluyendo especies introducidas. Para el género Opuntia, House y Midence (2007) reporta cinco espe- cies. Y según Nelson (2008), el género Opuntia está representado en Honduras por seis especies nativas.

Esta investigación tiene como objetivo actualizar la taxonomía del género Opuntia para Honduras, incluyendo información sobre su diversidad, distribución y estado de conservación (CITES y UICN).

\section{Materiales y métodos}

\section{Área de estudio}

Este estudio fue realizado en la Republica de Honduras, América Central, país separado por tres regiones montañosas sierra del norte, cordillera central y el sistema montañoso del sur, ubicado entre las coordenadas $14^{\circ} 05^{\prime} 39^{\prime \prime}$ $\mathrm{N}, 87^{\circ} 12^{\prime} 24^{\prime \prime} \mathrm{W}$, con elevaciones que van desde los 0 hasta los $2870 \mathrm{~m}$, con temperatura anuales que oscilan entre los 21 y $32^{\circ} \mathrm{C}$ y con precipitaciones anuales desde los 1000 hasta los 2500 $\mathrm{mm}$.

\section{Métodos}

Se realizó la revisión de los ejemplares de Opuntia depositados en los dos herbarios principales de Honduras, herbario Cyril Hardy Nelson Sutherland (TEFH) de la Universidad Nacional Autónoma de Honduras y el herbario Paul C, Stanley (EAP) de la Universidad Zamorano. Se realizaron descripciones diagnosticas de las especies, analizando caracteres morfológicos considerados de importancia taxonómica o relevantes para su determinación. Además, se tomó nota de los sitios de recolecta, fechas de fenología, hábitat y nombres comunes.

Simultáneamente, se realizó una revisión detallada de la literatura especializada reciente de la zona (Véliz, 2008; Cerén et al. 2015; Bravo y Arias, 2011), para fortalecer la diagnosis de cada especie. Asimismo, se acompañó de información obtenida de bases de datos digital, como Trópicos del Missouri Botanical Garden (http://www.tropicos.org), Global Biodiversity Information Facility (https://www.gbif.org), Angiosperm Phylogeny Website, versión 14. (http://www.mobot.org/MOBOT/research/ 


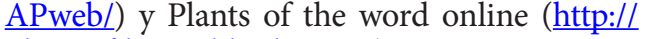
plantsoftheworldonline.org).

Los caracteres fueron analizados e incorporados en una clave taxonómica para la identificación de las especies del género Opuntia. Además, las descripciones se acompañan de ilustraciones realizados a partir de ejemplares de herbario y fotografías tomadas en campo. Para representar la distribución de las especies se elaboraron mapas en QGIS 2.8.

Finalmente, se revisó el estado de conservación de cada especie según la lista roja de la UICN (Unión Internacional para la Conservación de la Naturaleza) en https://www. iucnredlist.org y especies CITES (Convención sobre el Comercio Internacional de Especies Amenazadas de Fauna y Flora Silvestres) en http://checklist.cites.org.

\section{Resultados}

En total se revisaron 49 ejemplares de herbario, 30 en EAP y 19 en TEFH, reportándose ocho especies nativas del género Opuntia para Honduras (de las 12 que se registran para todo Centroamérica), donde Opuntia hondurensis es la única especie endémica para el país; $O$. deamii, O. decumbens, O. dejecta, O. guatemalensis, O. lutea y $O$. velizii son endémicas de Mesoamérica y Opuntia pubescens especie con amplia distribución en el Conteniente Americano. Las ochos especies registradas se distribuyen en 11 de los 18 departamentos de Honduras, donde Opuntia lutea es la especie con mayor distribución, reportándose en 5 departamentos. Por otro lado, O. pubescens y $O$. velizii son dos especies que están representadas por un solo ejemplar de herbario. Las ocho especies habitan en el bosque tropical seco, además, $O$. deamii, O. decumbens, O. guatemalensis y O. pubescens presentan afinidad por los suelos rocosos.

En cuanto al estado de conservación, estas ocho especies están protegidas por CITES dentro del Apéndice II, debido a su comercialización ilegal. Además, Opuntia decumbens, O. guatemalensis y O. pubescens se encuentran en la lista roja de la UICN en la categoría LC (preocupación menor), O. dejecta y O. lutea en la categoría DD (datos insuficientes), y O. deamii, $O$. hondurensis y $O$. velizii no presentan datos de conservación.

A continuación, se presenta la sinopsis taxonómica de Opuntia en Honduras, una clave de determinación, fotografías de las especies (Figura 1), descripciones, distribución, datos de floración y estatus de conservación para cada especie.

\section{Clave de identificación de las especies de Opuntia}

1. Plantas con tallos teretes............... pubescens

1 '. Plantas con tallos aplanados.

2. Plantas postradas: cladodios pubescentes; aréolas circundadas por una pigmentación rojiza-purpura. O. decumbens

2'. Plantas erectas; cladodios glabros; areolas sin pigmentación..

3. Aréolas de los cladodios con cerdas y espinas rígidas.

O. lutea

3 '. Aréolas de los cladodios sin cerdas pero con espinas rígidas o flexibles.

4. Flor en roseta, con los tépalos extendidos en la antesis, estambres insertos.

4 '. Flor tubular, con los tépalos erectos en la antesis, estambres exsertos.

5. Cladodios obovados, espinas de 4.5 a $5 \mathrm{~cm}$; flores con tépalos externos amarillos a rojizos... O. deamii

5'. Cladodios redondeados, espinas de hasta $3.5 \mathrm{~cm}$; flores con tépalos externos amarilloverdosos. O. guatemalensis

6. Cladodios con manchas azuladas; flores amarillas con matices rojo-anaranjado, de $5 \mathrm{~cm}$ hasta $8 \mathrm{~cm}$ de longitud; frutos claviformes

O. velizii

6'. Cladodios sin manchas azules; flores rojizas o rosados, de hasta $5 \mathrm{~cm}$ de longitud; frutos ovoides

7. Cladodios de hasta $15 \mathrm{~cm}$ de longitud; espinas de hasta $4 \mathrm{~cm}$ de longitud; flores de hasta 5 $\mathrm{cm}$ de longitud (incluido el estilo).....O. dejecta 

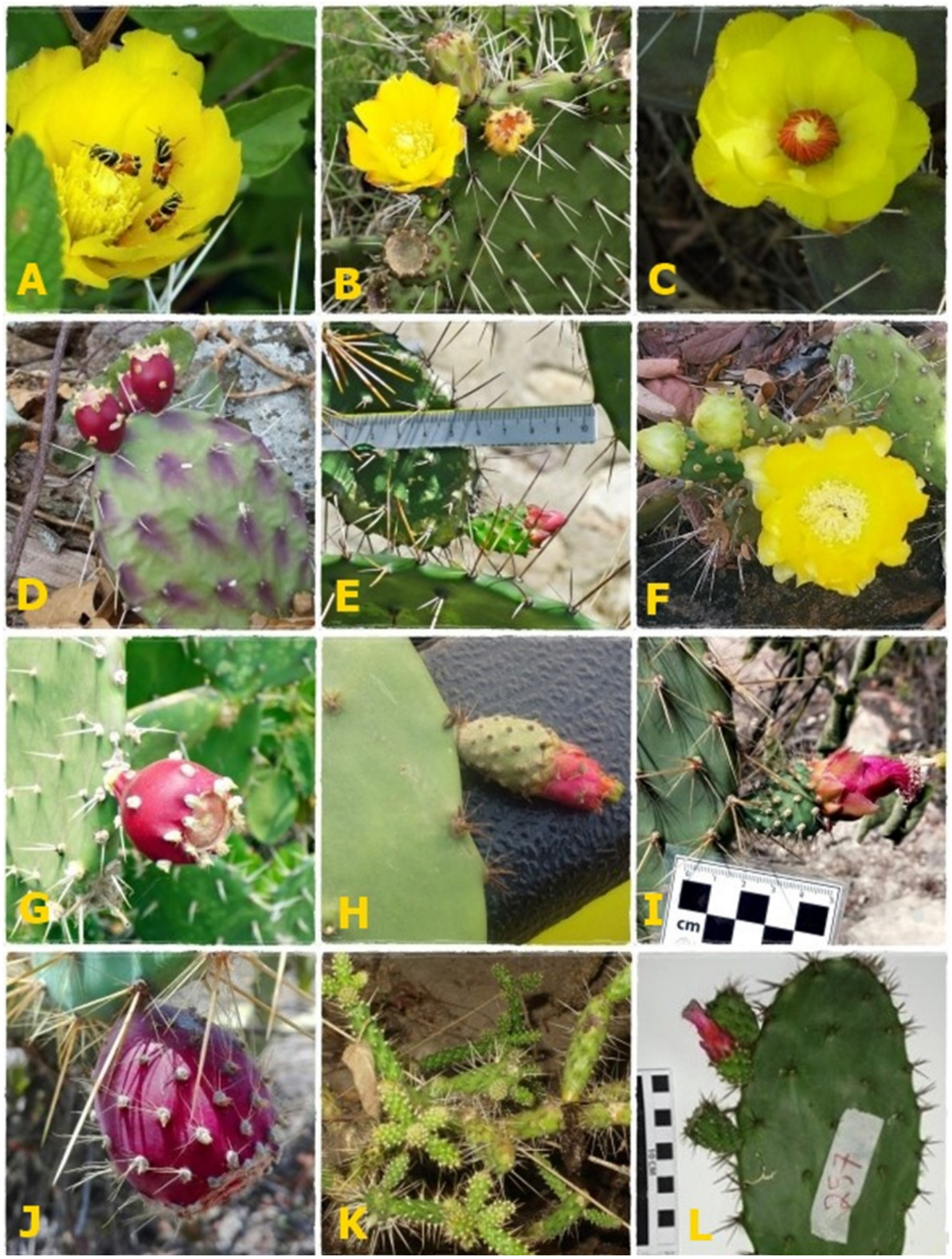

Figura 1. Especies de Opuntia en Honduras. A-B. O. deamii, C-D. O. decumbens, E. O. dejecta, F-G. O. guatemalensis, H. O. hondurensis, I-J. O. lutea, K. O. pubescens, L. O. velizii (Fotografías A, B, I y J de Olga Pineda; C, D, E, F, G, H y L de Olvin Oyuela; y K de Paul House). 
$7^{\prime}$. Cladodios mayores a $20 \mathrm{~cm}$ de longitud; espinas de hasta $2 \mathrm{~cm}$ de longitud; flores de $3 \mathrm{~cm}$ de longitud (incluido el estilo)....O. hondurensis

\section{Descripción botánica de las especies de Opuntia}

1. Opuntia deamii Rose. Contr. U.S. Natl. Herb. 13(9): 309, t. 65. 1911. TIPO: GUATEMALA. Fiscal, in open pasture along a ravine, 07 Nov 1909, C. C. Deam 6228 (Holotipo: US; Isotipos: $\mathrm{CAS}, \mathrm{MICH}, \mathrm{MO})$.

Nombre común: Tuna (zona central), caitillo (suroriental).

Descripción: Planta arbustiva, 0.7-1 $\mathrm{m}$ de alto; tallos ramificados, verde brillante, glabros, tronco cilíndrico y corto. Cladodios obovados a oblongos, de 13-25 cm de longitud y 7.5-16 $\mathrm{cm}$ de ancho. Aréolas pequeñas separadas entre sí por 3-4 cm. Espinas 2-4 aplanadas, extendidas, blancas o amarillentas, de $4-5 \mathrm{~cm}$ de longitud. Flores abiertas en la antesis con tépalos amarillos a rojizos, de $4-6 \mathrm{~cm}$ de longitud. Fruto en baya, ovoide o globoso, de 3.5-5 cm de longitud, rojizo-púrpura (Fig. 2).

Floración: febrero a junio.

Hábitat: Bosque seco tropical, suelos rocosos y en acantilados.

Distribución: Ocotepeque, Comayagua, Francisco Morazán y El Paraíso (Fig. 3). II).

Estado de conservación: CITES (Apéndice

Material examinado: HONDURAS. Comayagua, El Banco, $640 \mathrm{msnm}, 14^{\circ} 41^{\prime} 07^{\prime \prime} \mathrm{N}$, 8757'07" W, 28 Mar 1945, J. Valerio R. 2597 (EAP!); El Banco, 640 msnm, 14²41'07" N, 87 $57^{\circ} 07^{\prime \prime}$ W, 28 Mar 1945, J. Valerio R. 2598 (EAP!). El Paraíso, Llano Lizapa/Río Yaguare, $950 \mathrm{msnm}, 14^{\circ} 00^{\prime} \mathrm{N}, 87^{\circ} 00^{\prime} \mathrm{W}, 17$ May 1951, A. Molina 3957 (EAP!). Francisco Morazán, El Zamorano, 800-850 msnm, 14²41'32" N, 8757'48" W, 30 Jul 1949, P. C. Standley 22009 (EAP!); Las Mesas, $900 \mathrm{msnm}, 15$ Oct 1951, P. C. Standley 28875 (EAP!); Las Mesas, 30.VI.1961, L. J. Eilers 735 (EAP!); Tegucigalpa, $1000 \mathrm{msnm}, 14^{\circ} 02^{\prime} \mathrm{N}, 87^{\circ} 12^{\prime} \mathrm{W}, 11$ Sep 2005 , O. Almendares 81 (TEFH!); Sabana Grande.
1300 msnm, $13^{\circ} 54^{\prime} \mathrm{N}, 87^{\circ} 14^{\prime} \mathrm{W}, 10$ May 2016, L. Ferrufino et al. 717 (TEFH!). Ocotepeque, Belén Gualcho, 1500 msnm, 29 Jun 1976, C. Nelson et al. 3730 (TEFH!).

2. Opuntia decumbens Salm-Dyck. Hort. Dyck. 361. 1834. TIPO: MÉXICO. “Tierra caliente", W. Karwinski s/n (Neotipo designado por Crook y Mottram 1996: Fitch W. H. en Hooker W. J. 1841. Curtis's Botanical Magazine 68: pl. 3984).

Sinónimos: Opuntia decumbens var. scheinvariana E. Paniagua, O. heliae Matuda

Nombre común: Tuna (zona central), lengua de vaca (zona occidental), caitillo (zona suroriental).

Descripción: Planta arbustiva baja postrada de $0.20-1 \mathrm{~m}$ de alto; tronco no definido. Cladodios obovados, elípticos o angostamente ovados, de $8-13 \mathrm{~cm}$ de longitud y $5-7 \mathrm{~cm}$ de ancho, pubescentes. Aréolas circundadas por una pigmentación rojiza (Fig. 4), separadas entre sí por 1-2.3 cm. Espinas 2-3 delgadas, grises o amarillas, de $4-8 \mathrm{~cm}$ de longitud. Flores abiertas en la antesis, con tépalos amarillos, son los segmentos del perianto de color rojizo, de $3-6 \mathrm{~cm}$ de longitud. Fruto en baya, globosos, de $2-3 \mathrm{~cm}$ de longitud, pubescente, rojo púrpura.

Floración: noviembre a mayo.

Hábitat: Bosque seco tropical, suelos rocosos y cercos de rocas.

Distribución: Francisco Morazán, El Paraíso y La Paz (Fig. 5).

Estado de conservación: CITES (Apéndice II) y UICN (LC).

Material examinado: HONDURAS. El Paraíso, Yuscarán, $500 \mathrm{msnm}, 14^{\circ} 21^{\prime} \mathrm{N}, 8^{\circ} 13^{\prime}$ W, 7 Oct 1994, J. L. Linares y R. L. Metsger 1736 (EAP!). Francisco Morazán, San Antonio de Oriente, Región el Chahuite. 800-1075 msnm, 5 Mar 1947, P. C. Standley et al. 5116 (EAP!); Sabanagrande, $835 \mathrm{msnm}, 13^{\circ} 46^{\prime} \mathrm{N}, 87^{\circ} 13^{\prime} \mathrm{W}$, 10 May 2016, L. Ferrufino et al. 718 (TEFH!). La Paz, El Llano de las Lagunas, $700 \mathrm{msnm}, 8$ Feb 1983, B. K. Holst 838 (EAP!, TEFH!). 


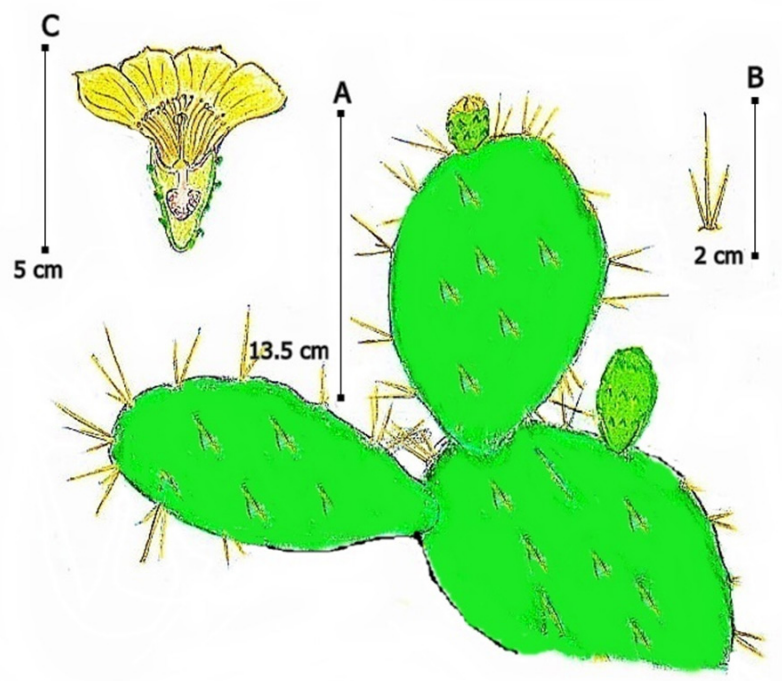

Figura 2. Opuntia deamii. A. Cladodio terminal; B. Areola y espinas; C. Flor en corte longitudinal.

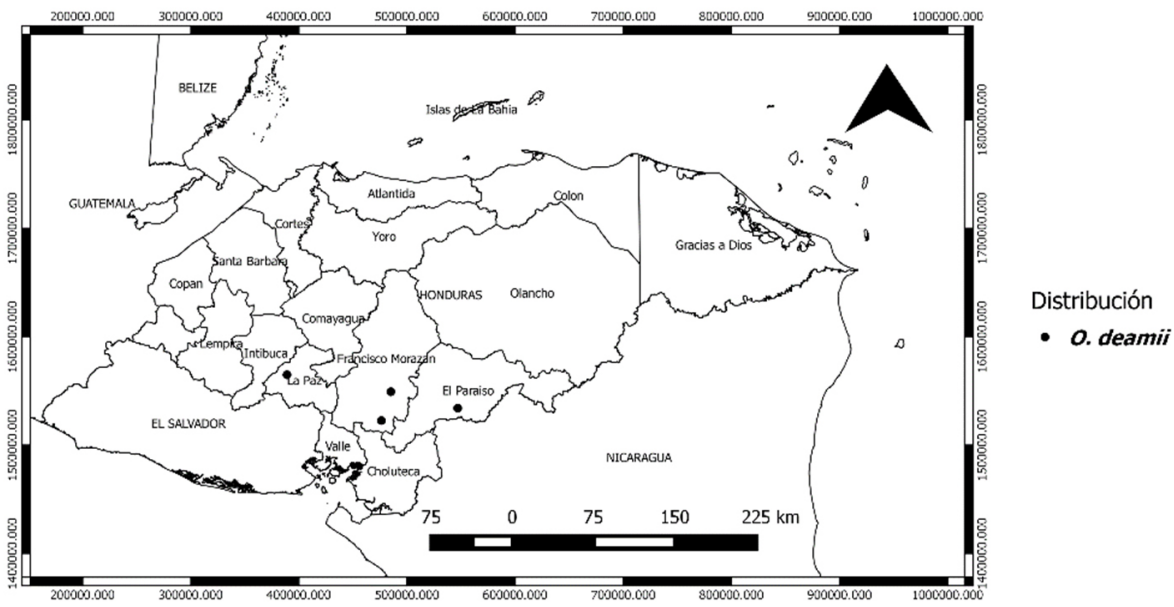

Figura 3. Distribución de O. deamii en Honduras, basado ejemplares del herbario EAP y TEFH.

3. Opuntia dejecta Salm-Dyck. Hort. Dyck. 361. 1834. Nopalea dejecta (Salm-Dyck) Salm-Dyck, Cact. Hort. Dyck. 64.1849 [1850]. TIPO: CUBA. Habana, sin localidad ni espécimen citados (para más detalles ver Britton y Rose 1919: 37).

Sinónimos: Nopalea escuintlensis Matuda
Nombre común: Tuna (zona central), nopal (zona central).

Descripción: Planta arbustiva ramificada, de 1-3 m de alto; tronco definido, con ramificación ascendente y difusa. Cladodios lanceolados, angostos, de $8-13.5 \mathrm{~cm}$ de longitud y 3-5 cm de ancho, glabros. Aréolas separadas 


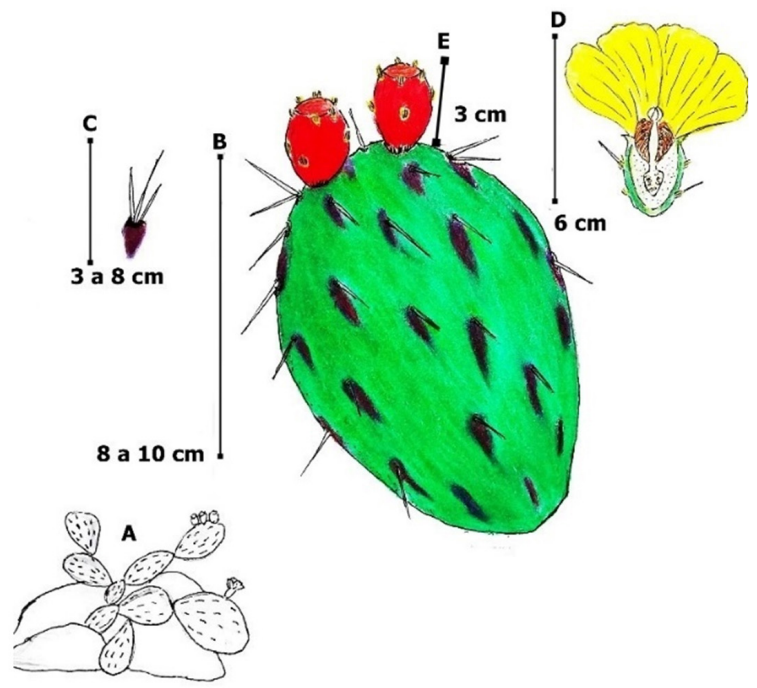

Figura 4. Opuntia decumbens. A. Hábito de crecimiento; B. cladodio terminal con manchas moradas; C. Areola y espinas; D. Flor en corte longitudinal; E. fruto en baya.

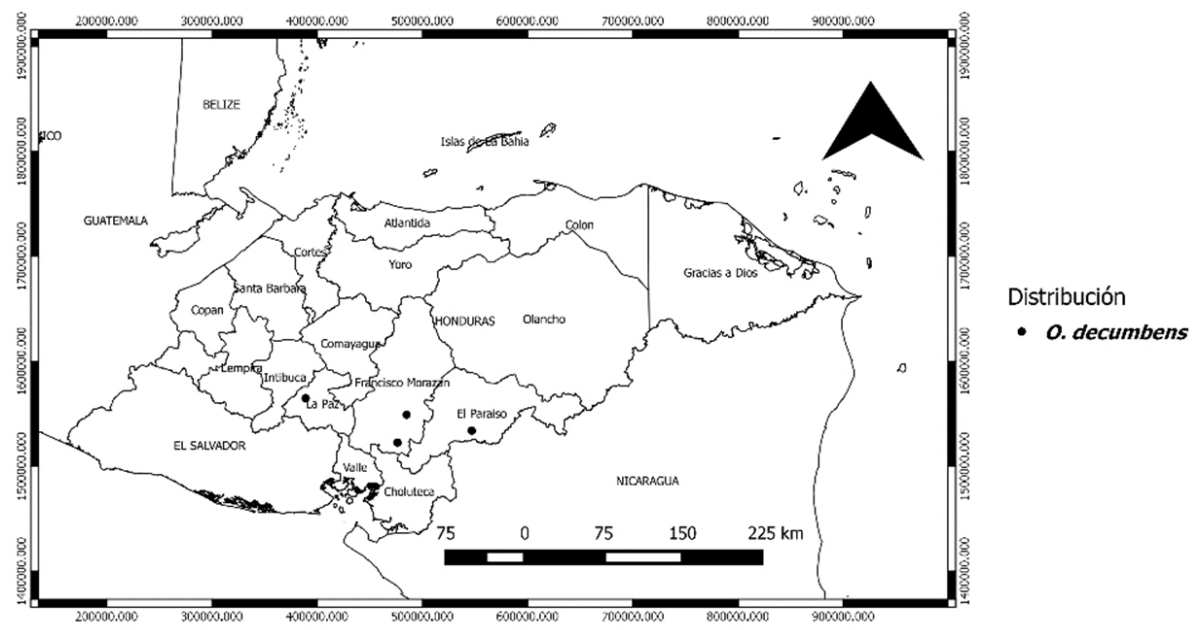

Figura 5. Distribución de O. decumbens en Honduras, basado ejemplares del herbario EAP y TEFH.

entre sí por $0.8-2.2 \mathrm{~cm}$. Espinas $2-3$ rojizas o anaranjadas, de $2-3 \mathrm{~cm}$ de longitud. Flores tubulares rosadas a rojizas (Fig. 6), de $4-5 \mathrm{~cm}$ de longitud. Fruto en baya, globoso, de 3-4 cm de longitud, liso, con numerosas gloquidios, rojo oscuro.

Floración: octubre a mayo.
Hábitat: Bosque seco tropical, suelos calcáreos.

Distribución: El Paraíso y Lempira (Fig. 7).

Estado de conservación: CITES (Apéndice II) y UICN (DD).

Material examinado: HONDURAS. El 


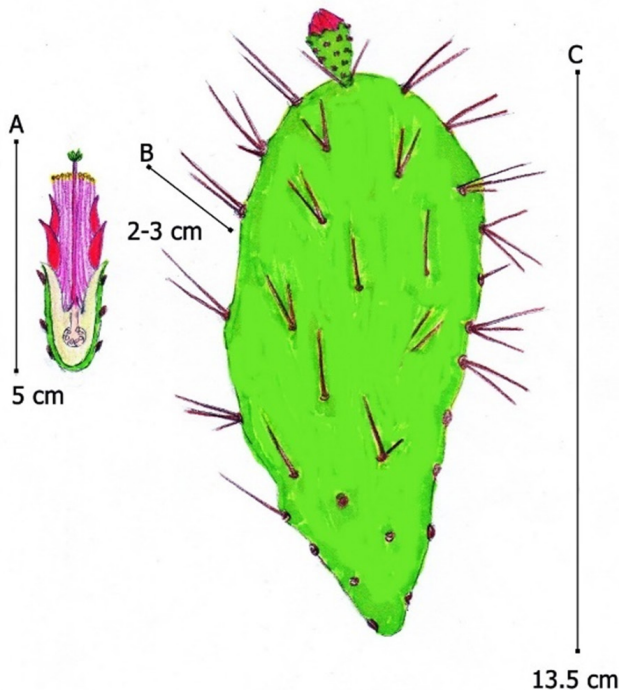

Figura 6. Opuntia dejecta. A. Flor en corte longitudinal; B. Areola y espinas; C. Cladodio terminal con botón floral.

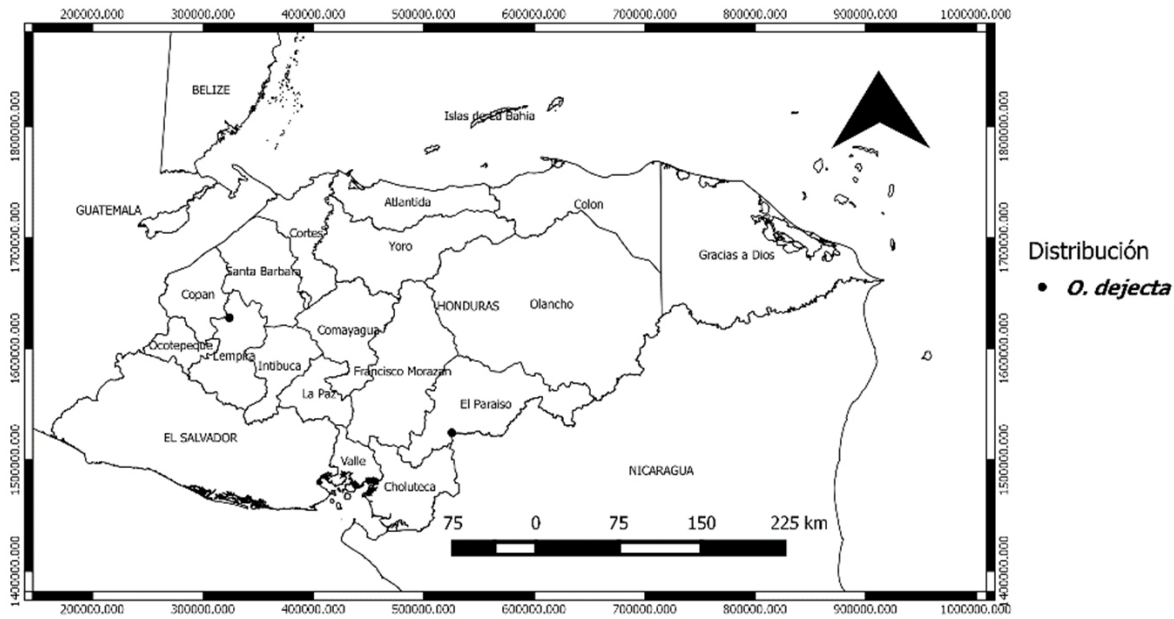

Figura 7. Distribución de O. dejecta en Honduras, basado ejemplares del herbario EAP y TEFH.

Paraíso, Río Choluteca, 600 msnm, 27 Mar 1947, P. C. Standley 4959 (EAP!). Lempira, Las Flores, $581 \mathrm{msnm}, 14^{\circ} 43^{\prime} \mathrm{N}, 88^{\circ} 38^{\prime} \mathrm{W}$, $21 \mathrm{Jul}$ 2016, O. Oyuela et al. 002 (EAP!, TEFH!).

4. Opuntia guatemalensis Britton \& Rose. Cact. 1: 218, f. 285. 1919. TIPO: GUATEMALA. Sin localidad, 1909, G. B. Wilcox s/n (Holotipo: US; Isotipo: NY).
Sinónimos: Opuntia salvadorensis Britton \& Rose

Nombre común: Tuna (zona central), caitillo (suroriental).

Descripción: Planta arbustiva, de $0.40-1.20$ $\mathrm{m}$ de alto; con ramificación basal ascendente. Cladodios obovados, de 6 - $17 \mathrm{~cm}$ longitud y 


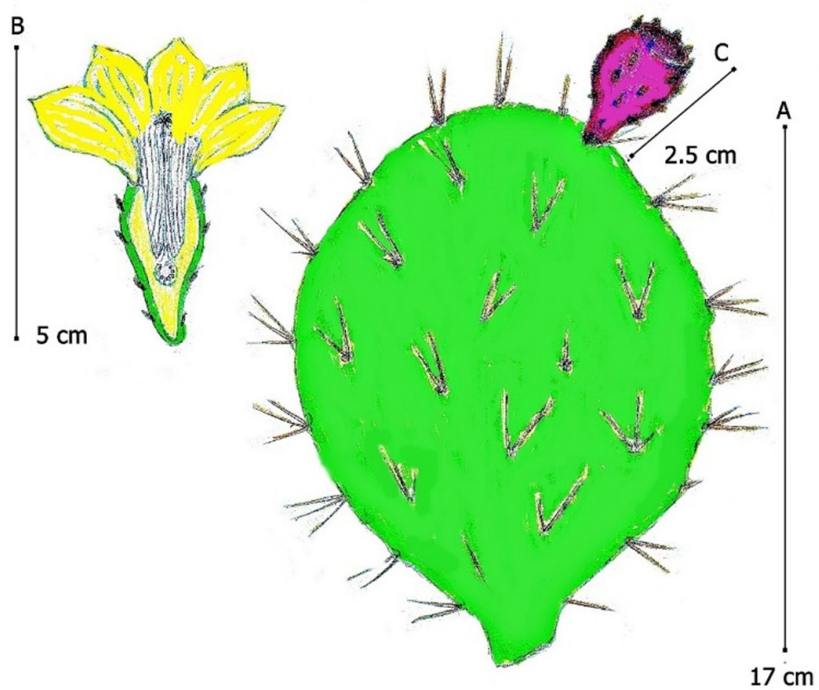

Figura 8. Opuntia guatemalensis. A. Cladodio terminal; B. Flor en corte longitudinal; C. Fruto.

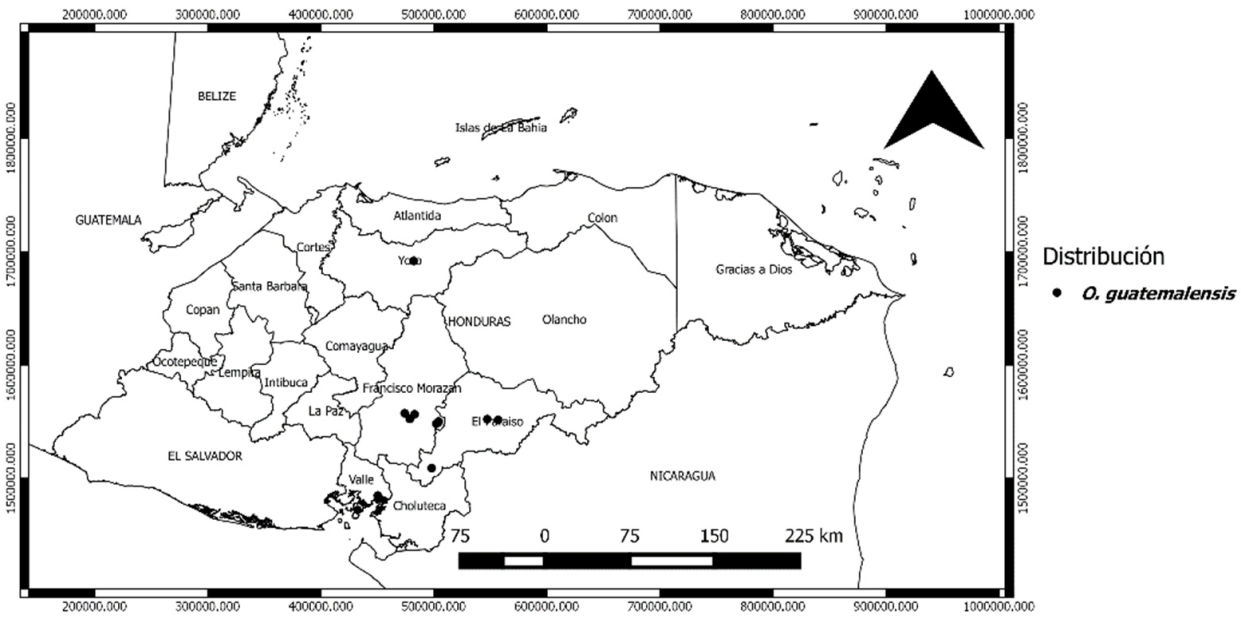

Figura 9. Distribución de O. guatemalensis en Honduras, basado ejemplares del herbario EAP y TEFH.

5-11.5 cm de ancho. Aréolas separas entre sí por $1.5-2.3 \mathrm{~cm}$. Espinas 1-3 reflexas, a veces ausentes, grises, blancas o amarillas, de 1.5-3.7 $\mathrm{cm}$ de longitud. Flores abiertas en la antesis con tépalos amarillos (Fig. 8), segmentos del perianto amarillo-verdosas de $5 \mathrm{~cm}$ de longitud. Frutos baya, ovoides, $2-2.8 \mathrm{~cm}$ de longitud, rojos a rojo-purpúreos.
Floración: enero a abril.

Hábitat: Bosque seco tropical, suelos rocosos y cercos de rocas.

Distribución: Comayagua, Francisco Morazán, El Paraíso, Valle y Yoro (Fig. 9).

Estado de conservación: CITES (Apéndice II) y UICN (LC). 


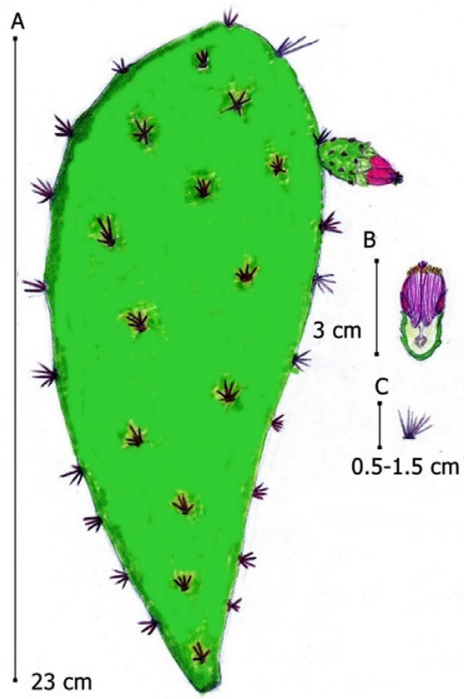

Figura 10. Opuntia hondurensis. A. Cladodio terminal; B. Flor en corte longitudinal; C. Areola y espinas.

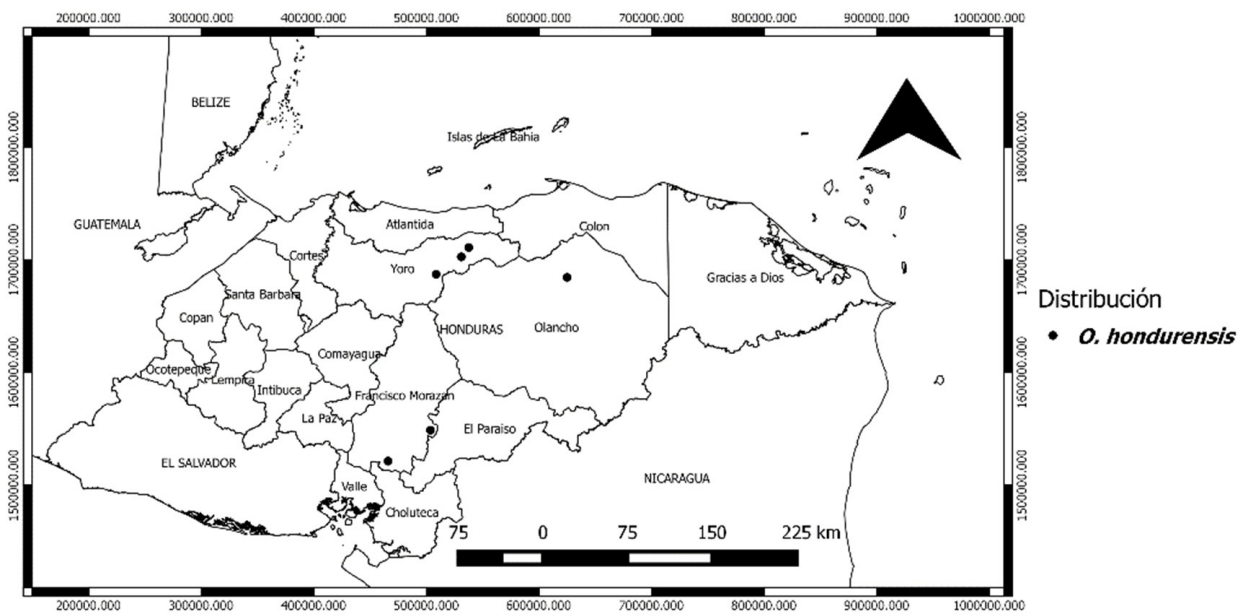

Figura 11. Distribución de O. hondurensis en Honduras, basado ejemplares del herbario EAP y TEFH.

Material examinado: HONDURAS. El Paraíso, Ojo de Agua, Región Quebrada de Dantas, 750 msnm, 11 Jul 1946, P. C. Standley 1303 (EAP!); Danlí, Rio San Cristobal, 25 Feb 1952, M. C. Carlson 2606 (EAP!); Texiguat, 320 msnm, $13^{\circ} 39^{\prime} \mathrm{N}, 87^{\circ} 01^{\prime}$ W, 10 May 2016, L. Ferrufino et al. 725 (TEFH!). Francisco Morazán, Distrito Central, Colonia Suyapa, $1200 \mathrm{msnm}$, 25 Nov 1946, P. C. Standley y L. O. Williams
4065 (EAP!); Distrito Central, Residencial La Fuente, 950 msnm, 9 May 1984, R. Paz 185 (TEFH!); San Antonio de Oriente, El Zamorano, 780 - 900 msnm, 17 Feb 1947, P. C. Standley 4958 (EAP!); El Zamorano, $800 \mathrm{msnm}$, $14^{\circ} 00^{\prime} \mathrm{N}, 87^{\circ} 00^{\prime} \mathrm{W}, 14$ May1948, L. O. Wilson y A. Molina 14193 (EAP!); Las Mesas, 800-900 msnm, $14^{\circ} 28^{\prime} \mathrm{N}, 89^{\circ} 00^{\prime} \mathrm{W}, 3$ Sep 1949, P. C. 


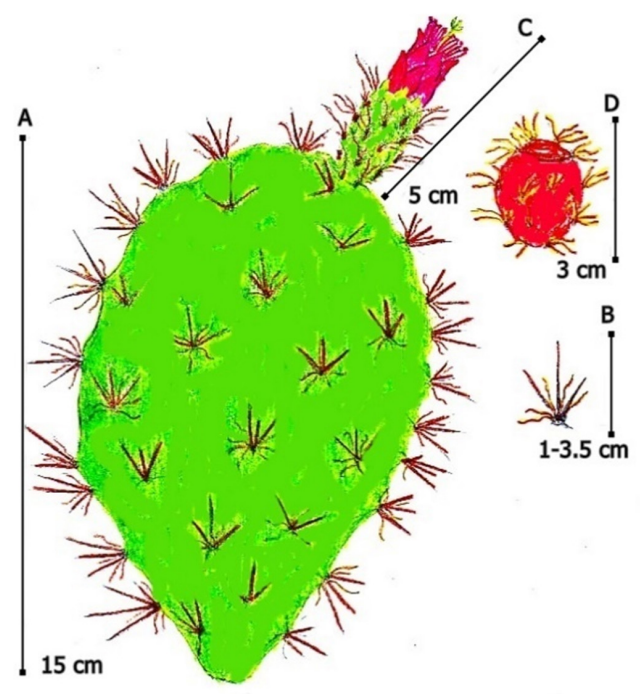

Figura 12. Opuntia lutea. A. Cladodio terminal; B. Areola con espinas rigidas y cerdas; C. Flor; D. fruto con cerdas.

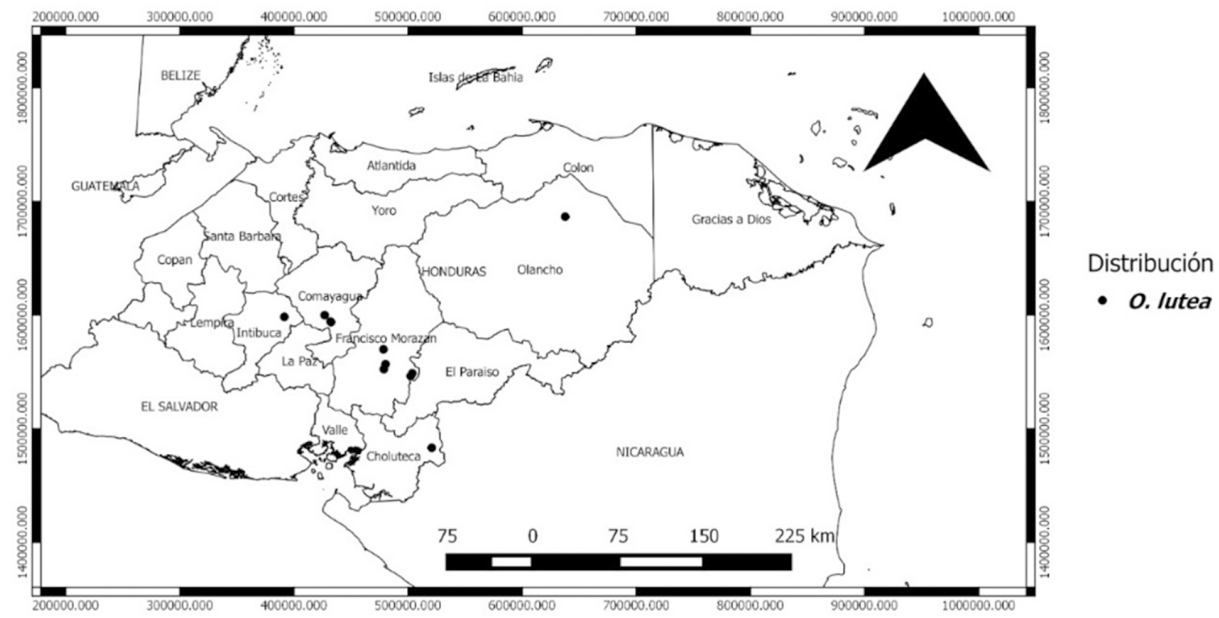

Figura 13. Distribución de O. lutea en Honduras, basado ejemplares del herbario EAP y TEFH.

Standley 23374 (EAP!). Valle, Desvío a Coyolito, 2 Abr 1977, Gladys Yong 126 (TEFH!); San Lorenzo, Puerto Soto, 23 ene 1974, C. Nelson 1479 (TEFH!). Yoro, sin localidad, R. Zúniga 009 (TEFH!).

5. Opuntia hondurensis Standl. Publ. Field Mus. Nat. Hist., Bot. Ser. 9(4): 316. 1940. Nopalea hondurensis (Standl.) Rebman, J. Arizo-
na-Nevada Acad. Sci. 34(1): 45. 2002. TIPO: HONDURAS. Yoro, vecinity of Coyoles, in the Aguan River valley, 27 Jun 1938, T. G. Yuncker et al. 8258 (Holotipo: F; Isotipo: BM, G, GH, MICH, NY, S, US).

Nombre común: Tuna (zona central), oreja de vaca (zona occidental) y nopales (zona norte). 
Descripción: Planta arborescente de 7-9 m de alto; tronco de $30 \mathrm{~cm}$ de diámetro. Cladodios ovados-oblongos, de $13-26 \mathrm{~cm}$ de longitud y 6-9 $\mathrm{cm}$ de ancho. Aréolas separas entre sí por $2-3.3 \mathrm{~cm}$. Espinas $4-6$ rojizas, de $0.5-1.5 \mathrm{~cm}$ de longitud. Flores rosado rojizas, tubulares (Fig. 10 ), de hasta $3 \mathrm{~cm}$ de longitud. Fruto ovoide, de $2.5-3 \mathrm{~cm}$ de longitud, rojo-purpúreos.

Floración: febrero a junio.

Hábitat: Bosque seco tropical, suelos de valle areniscos.

Distribución: Francisco Morazán (cultivado), Yoro y Olancho (Fig. 11). II)

Estado de conservación: CITES (Apéndice

Material examinado: HONDURAS. Francisco Morazán, La Venta, 700 msnm, 14 Feb 1971, A. Molina 25929 (EAP!); San Antonio de Oriente, El Zamorano, 800 msnm, 6 Dic 1984, A. Molina 33858 (EAP!). Olancho, Botadero, 700 msnm, $15^{\circ} 14^{\prime} \mathrm{N}, 85^{\circ} 50^{\prime} \mathrm{W}, 4$ Feb $2015, \mathrm{~S}$. Cruz et al. 194 (TEFH!). Yoro, Los Encuentros, $289 \mathrm{msnm}, 15^{\circ} 15^{\prime} \mathrm{N}, 86^{\circ} 55^{\prime} \mathrm{W}, 11$ May 2016, L. Ferrufino et al. 735 (TEFH!); San José, 200 msnm, 1528'30" N, 86³9'01" W, 2 Jul 1994, G. Davised et al. 35 (EAP!).

6. Opuntia lutea (Rose) D.R. Hunt. Cactaceae Consensus Init. 4: 6. 1997. Nopalea lutea Rose. Contr. U.S. Natl. Herb. 12(9): 405, t. 58. 1909. TIPO: GUATEMALA. Jalapa, near El Rancho, alt. 300 m, 28 Dic 1907, W. A. Kellerman 7046 (Holotipo: US; Isotipo: MO).

Nombre común: Tuna (zona central), nopal (zona central).

Descripción: Planta arbustiva o arborescente, de 5-7 $\mathrm{m}$ de alto; tronco corto bien definido. Cladodios obovados a oblongos, de 13-23 $\mathrm{cm}$ de longitud y $5-11 \mathrm{~cm}$ de ancho. Aréolas de 3-7 mm, distantes entre sí por $1.3-2 \mathrm{~cm}$. Espinas 3-6, aciculares, rectas y rígidas, con cerdas amarillas a rojizo, de $1-3.5 \mathrm{~cm}$ de longitud. Flores tubulares con tépalos rosado-rojizos, de $4-5 \mathrm{~cm}$ de longitud. Frutos baya, obovoide, de $2-3.5 \mathrm{~cm}$ de longitud, con numerosas gloquidios, rojos (fig. 12).
Floración: agosto a marzo.

Hábitat: Bosque seco tropical, áreas abiertas.

Distribución: Francisco Morazán, Comayagua, Intibucá, Olancho y El Paraíso (Fig. 13).

Estado de conservación: CITES (Apéndice II) y UICN (DD).

Material examinado: HONDURAS. Choluteca, San Marcos de Colón, La Mesita, 1200 msnm, 24 Ene 1974, C. Nelson 1383 (TEFH!). Comayagua, El Rosario, El Banco, $640 \mathrm{msnm}$, $15^{\circ} 02^{\prime} 49^{\prime \prime} \mathrm{N}, 87^{\circ} 45^{\prime} 43^{\prime \prime} \mathrm{W}, 28$ Mar 1945, J. V. Rodríguez 2599 (EAP!); Valle de Comayagua, 600 msnm, 6 Oct 1994, J. L. Linares y R. L. Metsger 1693 (EAP!); Valle de Comayagua, 600 msnm, $14^{\circ} 51^{\prime} 19^{\prime \prime} \mathrm{N}, 89^{\circ} 07^{\prime} 41^{\prime \prime} \mathrm{W}, 27$ Dic 1952, R. P. Williams y L. O. Williams 18829 (EAP!). Francisco Morazán, Cofradía, $860 \mathrm{msnm}, 1^{\circ} 22^{\prime}$ N, 87²1' W, 11 May 2016, L. Ferrufino et al. 729 (TEFH!); Santa Inés, 700 msnm, 135' N, $86^{\circ} 56^{\prime}$ W, 17 Jun 2016, T. López y D. Benítez s/n (TEFH!); Tegucigalpa, $1100 \mathrm{msnm}, 1^{\circ} 51^{\prime} 19^{\prime \prime}$ N, 8907'41" W, 18. Feb 1948, L. O. Wilson y A. Molina 13658 (EAP!); Colonia Florencia, $1050 \mathrm{msnm}, 14^{\circ} 05^{\prime} \mathrm{N}, 87^{\circ} 11^{\prime} \mathrm{W}, 5$ Mar 2005, N. Sahury Quan 113 (TEFH!); San Antonio de Oriente, El Zamorano, 800 msnm, 14²1'42" N, 87²41'56" W, 15 Nov 1945, J. V. Rodríguez 3595 (EAP!). Intibucá, Jesús de Otoro, $606 \mathrm{msnm}$, $14^{\circ} 27^{\prime}$ N, 8800' W, 27 Ago 2016, L. Ferrufino et al. 778 (TEFH!). Olancho, Aldea el Suyate, 600 msnm, 20 Ene 1988, M. L. Palacios 487 (TEFH!).

7. Opuntia pubescens H.L. Wendl. ex Pfeiff. Enum. Diag. Cact: 149. 1837. Cactus pubescens (H.L. Wendl. ex Pfeiff.) Lem. Cactées 87. 1868. TIPO: MÉXICO. Sin localidad ni especímenes citados (ver discusión para más detalles).

Sinónimos: Cactus nanus Kunth, Cereus nanas (Kunth) DC., Opuntia depauperata Britton \& Rose, Opuntia tayapayensis Cardenas, Platyopuntia nana (Kunth) F. Ritter.

Nombre común: Tuna (zona central).

Descripción: Planta arbustiva de hasta 0.6 $\mathrm{m}$ de alto; tallos con ramificación difusa o ascendente. Cladodios frágiles, cilíndricos-apla- 
nados, pubescentes, de $3-12 \mathrm{~cm}$ de longitud y 2-6 cm de ancho. Aréolas distantes entre sí por $0.4-0.8 \mathrm{~cm}$. Espinas 2-3, anaranjado-rojizas, de $0.5-2 \mathrm{~cm}$ de longitud. Flores amarillo-verdosas, de 4-6 cm de longitud. Frutos globosos baya, de $2-3 \mathrm{~cm}$ de longitud, morado-rojizos (Fig. 14).

\section{Floración: enero a marzo.}

Hábitat: Bosque seco tropical, suelos rocosos.

Distribución: Francisco Morazán (Fig. 15).

Estado de conservación: CITES (Apéndice II) y UICN (LC).

Material examinado: HONDURAS. Francisco Morazán, Río Hondo, $800 \mathrm{msnm}, 14^{\circ} 15^{\prime}$ N, 87²15' W, 5 Nov 2005, P. R. House 3196 (TEFH!).

8. Opuntia velizii Lodé. Cact.-Avent. Int. 102: 30, f. s/n. 2014. Nopalea guatemalensis Rose. Smithsonian Misc. Collect. 50: 330, t. 41, 42. 1907. TIPO: GUATEMALA. Jalape, El Rancho, alt. 270 m, 5 Abr 1905, W. R. Maxon 3774 (Holotipo: US).

Nombre común: Tuna (zona central).

Descripción: Planta arbustiva o arborescentes, de 2-5 m de alto; tallo principal no definido, con ramificación ascendente y difusa. Cladodios ovados hasta oblongos, verde-azulados, de $15-20 \mathrm{~cm}$ de longitud y 6 $-10 \mathrm{~cm}$ ancho. Aréolas distantes entre sí por $2.3-3.5 \mathrm{~cm}$. Espinas 5-8 desiguales, aciculares, rectas, rígidas, algunas flexibles, blancas o algo rosadas, de 5-7 $\mathrm{cm}$ de longitud. Flores tubulares rojas, hasta anaranjado-amarillas, de $5-8 \mathrm{~cm}$ de longitud. Frutos baya, claviformes a cónicos, de $4-5 \mathrm{~cm}$ de longitud (Fig. 16), rojo-purpúreos.

Fenología: julio a mayo.

Hábitat: Bosque seco tropical, sitios abiertos, orilla de cercos.

Distribución: El Paraíso (Fig. 15).

Estado de conservación: CITES (Apéndice II).

Material examinado: HONDURAS. El Paraíso, Río Choluteca, cerca de Ojo de
Agua, 600 msnm, 31 Jul 1950, P. C. Standley 28132 (EAP!).

\section{Discusión}

La taxonomía del género Opuntia se ha actualizado mediante estudios filogenéticos, al presente Opuntia incluye las especies del género Nopalea Salm-Dyck (Griffith y Porter 2009; Mercado 2014; Hunt 2016), a pesar de que sus morfologías florales sea muy distintivas (Mercado 2014). Siendo Nopalea reconocida por presentar flores tubulares y de color rosado a rojizas, mayormente polinizadas por picaflores, a diferencia de las del género Opuntia sensu stricto, con flores rotáceas amarillas, con tigmonastia en sus estambres, y polinizadas por insectos diurnos generalmente abejas (Ostolaza 2011; Lodé 2014). Al no considerarse esto suficiente para mantenerlos como géneros separados, Nyffeler y Eggli (2010) confirman la inclusión de Nopalea dentro de Opuntia, dejando al primero como sinónimo. El hecho de incluir a Nopalea en el género Opuntia, obligó a cambiar la nomenclatura de Nopalea guatemalensis ya que existía Opuntia guatemalensis como tal, por lo que para evitar confusión paso a nombrarse Opuntia velizii, en honor a Mario Véliz por Lodé (2014).

Esta sinopsis del género Opuntia es la primera que se hace para Honduras, aunque estudios anteriores han reportado especies de este género. House y Midence (2007) reportan ocho especies, cinco como Opuntia (O. deamii, O. decumbens, O. guatemalensis, O. pubescens y O. tomentella) y tres como Nopalea, (N. guatemalensis, $N$. hondurensis y N. lutea). Por su parte, Nelson (2008) reporta seis especies nativas, O. deamii, O. decumbens, O. guatemalensis, O. hondurensis, O. lutea y O. tomentella, esta última especie indicada como material en prensa por House y Midence (2007) y sin ningún ingreso a alguna colección de herbario. Asimismo, Bravo y Arias (2011) reportan siete especies, cuatro como Opuntia (O. deamii, O. decumbens, O. guatemalensis y O. pubescens) y tres como Nopalea (N. guatemalensis, N. lutea y N. dejecta). 


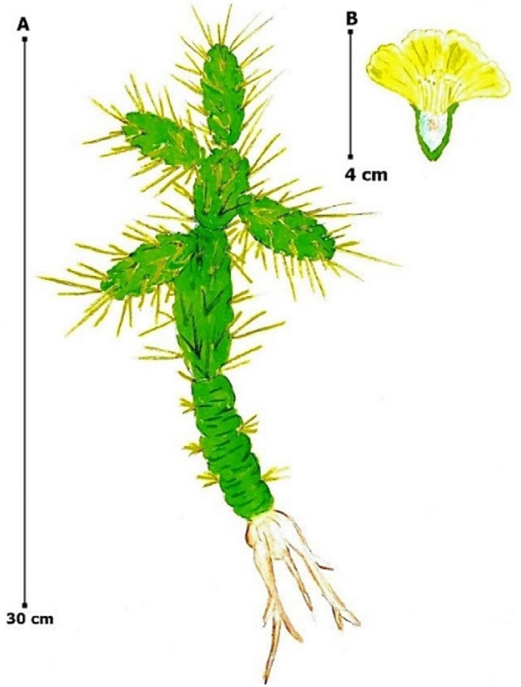

Figura 14. Opuntia pubescens. A. Planta en estado adulto; B. Flor en corte longitudinal.

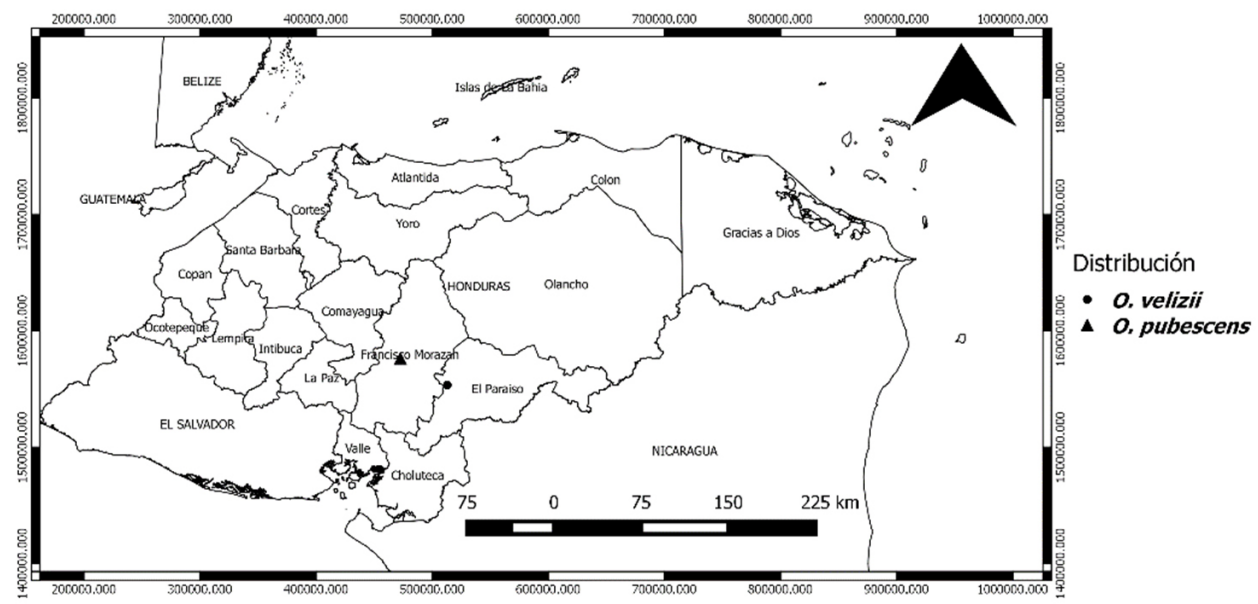

Figura 15. Distribución de O. pubescens y O. velizii en Honduras, basado ejemplares del herbario EAP y TEFH.

Debido a estos cambios taxonómicos, al registro de vouchers de herbario y de documentación precisa este estudio reconoce ocho especies nativas para Honduras (O. deamii, $O$. decumbens, O. dejecta, O. guatemalensis, $O$. hondurensis, O. lutea, O. pubescens y O. velizii). Por otro lado, no se reconoce a $O$. tomentosa (= O. tomentella) por la falta de especímenes de referencia y documentación.
Se reconoce que el género, al igual que la familia, no está bien representada en los herbarios revisados. Se observó una baja cantidad de muestras, la mayoría procedentes de la zona central del país haciendo notar que se han hecho muy pocos esfuerzos de recolecta. Esto puede deberse a la peculiar anatomía que presentan las cactáceas, en especial este género, haciéndolas difícil de herborizar y también al 


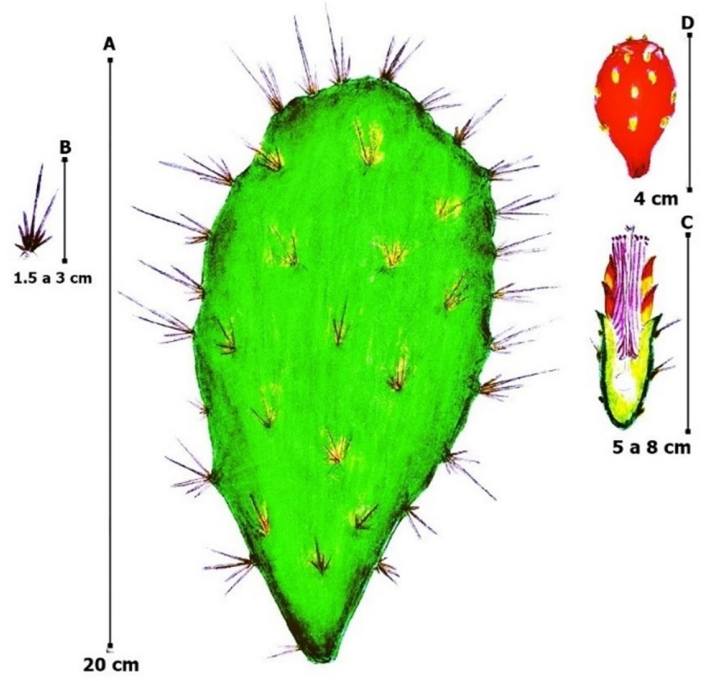

Figura 16. Opuntia velizii. A. Cladodio terminal; B. Areola y espinas; C. Flor en corte longitudinal; D. Fruto claviforme.

bajo interés en el estudio de estas (Pauca-Tanco y Quipuscoa-Silvestre 2017). Dejando la necesidad de realizar esfuerzos de recoleta y estudios poblacionales, para tener más ejemplares en los herbarios y más información, que pueda afianzar los datos para una clara comprensión de este género para Honduras.

La diversidad del género Opuntia en Honduras es incomparable con países como México donde este género está sumamente diversificado con aproximadamente 89 especies (Hunt 2016), pero en comparación con los países de la región centroamericana, la diversidad de especies del género Opuntia, solo es superada por Guatemala que reporta diez especies (Arias y Véliz 2006; Véliz 2008).

A pesar de que este género es tan reconocido y estudiado en América, aún existen carencias de información respecto al material tipo vinculado a cada especie. Por ejemplo, para O. dejecta Britton y Rose (1919) indican que el tipo ha sido erróneamente ubicado en Cuba y que no existe ningún espécimen. Algo similar ocurre con O. pubescens, donde solo se sabe que el tipo es de México pero en el protólogo no existe mención a ninguna localidad o espécimen. Por lo tanto ambas especies necesitarían estudios más detallados para realizar las tipificaciones necesarias.

En cuanto a la conservación de especies cabe destacar, que Opuntia y la familia en general, son plantas que se han adaptado a los suelos áridos y pedregosos, manteniendo el equilibrio dinámico de los ecosistemas, entre ellos del bosque seco tropical (Gurvich 2010; Jiménez 2011). En la actualidad los bosques secos se encuentran sometidos a una gran presión antrópica, poniendo en peligro su biodiversidad residente (Gentry 1992; Miles et al. 2006; Espinoza et al. 2012; Ferrufino et al. 2019), por ejemplo, las especies del género Opuntia, que se encuentran en estas zonas, son repudiados por los agricultores y ganaderos, debido a sus espinas y gloquidios, que puede lastimarlos tanto a ellos como al ganado. Aunque, al mismo tiempo sirven como una alternativa para el forrajeo de ganado en zonas áridas (López-Reyes et al. 2009). Por ello algunas especies de este género están amparadas dentro de UICN, que provee las medidas necesarias para proteger, conservar especies y disminuir la perdida de hábitat. También muchas especies son comercializadas ilegalmente, debido a esto el comercio de todas las especies de Cactaceae está regulado por las 
disposiciones de la convención CITES, quedando tres géneros actualmente exentos (Pereskia, Pereskiopsis Britton \& Rose y Quiabentia Britton \& Rose), al igual que híbridos y especies cultivadas. Todos los demás, incluido Opuntia, están sujetos al Apéndice II o en casos estrictos al Apéndice I (Hunt 2016).

\section{Conclusiones}

Se establece que el género en Honduras está representado por ocho especies. Siendo O. hondurensis la única especie endémica para el país, y con distribución natural en los departamentos de Yoro y Olancho, y cultivada en Jardines Botánicos de Francisco Morazán. Además, seis especies son endémicas para Mesoamérica, $O$. deamii, O. decumbens, O. dejecta, O. guatemalensis, O. lutea y O. velizii.

La distribución del género Opuntia en Honduras se da en el bosque tropical seco, en 11 departamentos del país, con Opuntia lutea como la especie con mayor distribución, reportada en cinco departamentos. En cambio, O. pubescens y $O$. velizii son especies que están representadas por un solo ejemplar de herbario. La falta de registros de herbario refleja la necesidad de ampliar esfuerzos de investigación. También se sugiere hacer exploraciones en busca de $O$. tomentosa para confirmar su presencia en el país, así mismo de O. pubescens y $O$. velizii para afianzar la información de estas especies.

Todas las especies del género Opuntia están incluidas en CITES dentro del apéndice II $\mathrm{y}$ algunas de las especies reportadas para Honduras están en la lista roja de la UICN en sus categorías LC (preocupación menor): Opuntia decumbens, O. guatemalensis y O. pubescens, y en la categoría DD (datos insuficientes): $O$. dejecta y O. lutea.

\section{Agradecimientos}

A Mario Blanco y Lilian Ferrufino por sus comentarios, correcciones y oportunas observaciones. A Rina Fabiola Díaz del Herbario EAP por su colaboración en la revisión del material de herbario.

\section{Bibliografía}

APG IV. 2016. An update of the Angiosperm Phylogeny Group classification for the orders and families of flowering plants: APG IV. Botanical Journal of the Linnean Society 181(1):1-20.

Arias, S; Véliz, M. 2006. Diversidad y distribución de las Cactaceae en Guatemala. Editor. E. Cano. Biodiversidad de Guatemala 1:229-238.

Arias, S. 2009. La familia Cactaceae. In A. Lot y Z. Cano-Santana (Eds.) Biodiversidad del Pedregal de San Ángel. UNAM, Reserva Ecológica del Pedregal de San Ángel y Coordinación de la Investigación Científica, D.F. México 135-141 p.

Arias, S; Gama, S; Vázquez, B; Guzmán L. 2012. Flora del Valle de Tehuacán-Cuicatlán. Cactaceae Juss. Instituto de Biología, Universidad Nacional Autónoma de México, México, D.F. $235 \mathrm{p}$.

Bravo, H. 1978. Las Cactáceas de México. Universidad Nacional Autónoma de México, México V. I. 743 p.

Bravo, H; Arias, S. 2011. Cactaceae en Flora Mesoamericana Vol. 2(1), (en línea). Consultado 20 Jun. 2019. Disponible en http:// legacy.tropicos.org/Name/40032719?projec$\underline{\text { tid=3\&langid }=66}$

Britton, N; Rose, J. 1919. The Cactaceae. Descriptions and illustrations of plants of the cactus family. New York Vol. I. 320 p.

Bueno, V; Plumed, J. 2017. Los Cactus. Monografías Botánica. Universidad de Valencia 1-173 p. ISBN: 978-84-9133-080-6.

Cerén, J; Menjivar, J; Arias, S. 2015. Diversidad de cactus de El Salvador. Museo de Historia Natural de El Salvador. San Salvador, El Salvador 1-152 p. ISBN 978-99923-908-5-6.

Crook, R; Mottram, R. 1996. Opuntia Index Part 2: Nomenclatural note and C-E. Bradleya 14(14):99-144. DOI: https://doi:10.25223/ brad.n14.1996.a15

Crook, R; Mottram, R. 1998. Opuntia Index Part 4: G-H. Bradleya 16(16):119-136. DOI: https://doi:10.25223/brad.n16.1998.a11 
De Almeida, O; de Souza, L; Paoli, A; Davis, A; Cota-Sánchez, J. 2018. Pericarp development in fruit of epiphytic cacti: implications for fruit classification and macro-morphology in the Cactaceae. Botany 96(9):621-635. DOI: $\underline{\text { https:// }}$ doi:10.1139/cjb-2018-0074

Espinosa, P; Vargas, A. 2002. Helia Bravo, pionera e inolvidable maestra. CONABIO. Biodiversitas 6(40):1-3.

Espinosa, C; de la Cruz, M; Luzuriaga, A; Escudero, A. 2012. Bosques tropicales secos de la región Pacífico Ecuatorial: diversidad, estructura, funcionamiento e implicaciones para la conservación. Ecosistemas 21(1-2):167-179.

Ferrufino, L; Cruz, S; Mejía, T; Rodríguez, F; Escoto, D; Sarmiento, E; Larkin, J. 2019. Composición, estructura y diversidad florística del bosque seco en el Valle de Agalta, Honduras. Madera y Bosques 25(2):1-15. DOI: http://dx. doi:10.21829/myb.2019.2521635

Fuentes-Pérez, M; Terrazas, T; Arias, S. 2009. Anatomía floral de cinco especies de Opuntia (Opuntioideae, Cactaceae) de México. Polibotánica 27:89-102.

Gentry, A. 1992. Tropical forest biodiversity: distributional patterns and their conservational significance. Oikos 63(1):19-28. DOI: http:// dx.doi.org/10.2307/3545512

Griffith, M; Porter, J. 2009: Phylogeny of Opuntioideae (Cactaceae). Int. J. Plant. Sci 170(1):107-116.

Gurvich, D. 2010. Registro de herbivoría de guanaco, Lama guanicoe, sobre Tephrocactus alexanderi (Cactaceae, Opuntioideae) en el Parque Provincial Ischigualasto (Prov. San Juan, Argentina). Boletín de la Sociedad Latinoamericana y del Caribe de Cactáceas y otras Suculentas 7(3):7-9.

House, P; Midence, C. 2007. El Bosque Seco, un reservorio genético natural para los cactus de Honduras. Revista Ciencia y Tecnología 1(II):79-94.

Hunt, D. R. 2016. CITES Cactaceae Checklist. Third Edition. Kew: Royal Botanic Gardens. 175 p. ISBN 978-0-9933113-2-1
Jiménez, C. 2011. Las cactáceas mexicanas y los riesgos que enfrentan. Revista Digital Universitaria 12(1):1-23.

Köhler, M; Font, F; Souza-Chies, T. 2018. First record of Opuntia rioplatense (Cactaceae) for the Brazilian Flora. Phytotaxa 379(4):293-296. DOI: https://doi:10.11646/phytotaxa.379.4.3

Las Penãs, M; Oakley, L; Moreno, N; Bernardello, G. 2017. Taxonomic and cytogenetic studies in Opuntia ser. Armatae (Cactaceae). Botany 95(2):101-120. DOI: https://doi.org/10.1139/ cjb-2016-0048

Lodé J. 2014. Adiós Nopalea. International Cactus-Adventures $N^{\circ}$ 104. España, 5 p.

Lodé J. 2015. Taxonomie des Cactaceae", La nouvelle classification des Cactées basée sur la recherche moléculaire et expliquée; Joël Lodé, Editions Cactus-Aventures Vol. 2: Maihuenia - Yungasocereus. ISBN: 978-84-617-3692-8. Disponible en https://cactusclassification.science/opuntia/

López-Reyes, M; Solís-Garza, G; Murrieta-Saldivar, J; López-Estudillo, R. 2009. Percepción de los ganaderos respecto a la sequía: viabilidad de un manejo de los agostaderos que prevenga sus efectos negativos. Estud. soc [online]. 17.221-241 p. ISSN 0188-4557.

Mercado, F. 2014. Diversidad y sistemática del género Opuntia s.s., en la región de los Cabos, Baja California Sur, México. Tesis Maestro en Ciencias. La Paz, B.C.S. México. Centro de Investigaciones Biológicas del Noroeste. 116 p.

Miles, L; Newton, A; DeFries, R; Ravilious, C; May, I; Blyth, S; Gordon, J. 2006. A global overview of the conservation status of tropical dry forests. Journal of Biogeography 33(3): 491-505. DOI: https://doi:10.1111/j.13652699.2005.01424.x

Nelson, C. 2008. Catálogo de Plantas Vasculares de Honduras, Spermatophytas. Editorial Guaymuras. Tegucigalpa, Honduras. 1576 p.

Nyffeler, R; Eggli, U.2010. A farewell to dated ideas and concepts: molecular phylogenetics and a revised suprageneric classification of the family Cactaceae. Schumannia 6:109-149. 
Ostolaza, C. 2011. 101 Cactus Del Perú. Lima. Ministerio del Ambiente. Perú. 253 p.

Pauca-Tanco, A; Quipuscoa-Silvestre, V. 2017. Catálogo de las cactáceas del departamento de Arequipa, Perú. Arnaldoa 24(2):447-96. DOI: http://doi.org/10.22497/arnaldoa.242.24204

Porras-Flórez, D; Albesiano, S; Arrieta-Violet, L. 2018. El género Opuntia (OpuntioideaeCactaceae) en el departamento de Santander, Colombia. Biota Colombiana 18(2):112-132. DOI: https://doi:10.21068/c2017.v18n02a07

Shulze, J. 2004. Elaboración de una guía ilustrada de Cactáceas en Honduras. Tesis de pregrado en Desarrollo Socioeconómico y Ambiente. El Zamorano, San Antonio de Oriente, Honduras. Escuela Agrícola Panamericana. 80 p.

Stevens, PF. 2001 en adelante. Angiosperm Phylogeny Website. Version 14, July 2017 [and more or less continuously updated since]. will do. Consultado 16 jun. 2020. Disponible en http://www.mobot.org/MOBOT/research/APweb/.

Stevens, W; Ulloa, C; Pool, A; Montiel, O. 2001. Flora de Nicaragua Publication: Monographs in Systematic Botany from the Missouri Botanical Garden 85: i-xlii. 1-2666.

Vázquez-Sánchez, M; Terrazas, T; Arias, S. 2012. El hábito y la forma de crecimiento en la tribu Cacteae (Cactaceae, Cactoideae). Botanical Sciences, 90(2):97-108 p. DOI: http://doi. org/10.17129/botsci.477

Véliz, M. 2008. Las Cactáceas de Guatemala. Litografías Modernas S. A. Guatemala. 130 p. 